\title{
Numerical Modeling of Smartphones with WCDMA, LTE, and WLAN Bands for Epidemiological Studies
}

\author{
Jisu Lee ${ }^{1} \cdot$ Ae-Kyoung Lee ${ }^{2}$ Seon-Eui Hong ${ }^{2} \cdot$ Hyung-Do Choi $^{2} \cdot$ Kyung-Young Jung ${ }^{1, *}$
}

\begin{abstract}
This paper presents the representative numerical modeling of smartphones with wideband code division multiple access (WCDMA), long-term evaluation (LTE), and wireless local area network (WLAN) bands for epidemiological studies. For this purpose, based on specific absorption rate (SAR) test reports for commercial smartphones released in South Korea from 2013 to 2019 , we determined the smartphone size, frequency band categorization, antenna locations, and target 1-g peak-spatial SAR (psSAR) values for a specific anthropomorphic mannequin (SAM) phantom. Numerical results showed that the designed numerical smartphone models yielded good matching and radiation performance, and more importantly, their 1-g psSAR values were within $\pm 16 \%$ of the target 1-g psSAR values.
\end{abstract}

Key Words: Finite-Difference Time-Domain Method (FDTD), Mobile Phone, Specific Absorption Rate (SAR), SAM Phantom.

\section{INTRODUCTION}

Epidemiological studies have actively analyzed the correlations between mobile phone use and the risk of brain tumors [1-4]. These cohort studies aimed to identify relationships between various risk factors and human health through follow-up research. However, cohort studies are costly and time-consuming, since they require long-term observation of changes in individual or group characteristics. Numerical specific absorption rate (SAR) studies have therefore been performed and have proven effective; for example, Wiart et al. [5] and Christ et al. [6] investigated electromagnetic (EM) exposure in the head tissues of children and adults using computational head phantoms and numerical handset models. Lee et al. [7] studied the relationship between brain SAR and various important factors, such as the mobile phone type, phone position, operating frequency, and user age using computational head phantoms and numerical mobile phone models. Additionally, Lee et al. [8] investigated brain SAR using computational average head models suitable for Korean males.

In numerical SAR studies, the development of numerical mobile phone models is highly important. In [7, 8], for epidemiological studies, the authors employed numerical mobile phone models [9] that were developed based on an investigation of commercial mobile phones released from 2002 to 2013 in South Korea. Note that the numerical mobile phone models in [9] were confined to $2 \mathrm{G}$ and $3 \mathrm{G}$ communication services. The evolutionary cycles of the mobile phone market and communication services have become shorter over time; therefore, these trends should be considered in numerical SAR studies of current mobile phones. Nowadays, smartphones are widely used for long-term evaluation (LTE) services.

Wireless local area network (WLAN) services are usually used for voice calls, texting, and web surfing; therefore, it is

Manuscript received April 27, 2021 ; Revised June 3, 2021 ; Accepted June 7, 2021. (ID No. 20210427-044J)

${ }^{1}$ Department of Electronic Engineering, Hanyang University, Seoul, Korea.

${ }^{2}$ Radio Technology Research Department, Electronics and Telecommunications Research Institute (ETRI), Daejeon, Korea.

"Corresponding Author: Kyung-Young Jung (e-mail: kyjung3@hanyang.ac.kr)

This is an Open-Access article distributed under the terms of the Creative Commons Attribution Non-Commercial License (http://creativecommons.org/licenses/by-nc/4.0) which permits unrestricted non-commercial use, distribution, and reproduction in any medium, provided the original work is properly cited.

(c) Copyright The Korean Institute of Electromagnetic Engineering and Science. 
important to develop numerical smartphone models for epidemiological studies that reflect the modern era, which is what this study has achieved.

By surveying SAR test reports provided by Samsung Electronics, we determined the smartphone size, frequency band categorization, antenna locations, and target 1 -g peak-spatial average SAR (psSAR) values for a specific anthropomorphic mannequin (SAM) phantom. We then developed numerical smartphone models with return losses larger than $6 \mathrm{~dB}$, radiation efficiencies greater than $70 \%$, and difference magnitudes of 1-g psSAR values between their target values below $20 \%$. All numerical simulations were performed using Sim4life [10], which produces simulations based on the accurate and powerful finite-difference time domain (FDTD) method [11-15].

\section{NUMERICAL SMARTPHONE MODELING}

In this study, we developed numerical smartphone models based on SAR test reports for smartphones distributed in South Korea from 2013 to 2019. First, we determined the size for the numerical smartphone models to be $73 \times 148 \times 8 \mathrm{~mm}^{3}$ by averaging the sizes of commercial smartphones provided in the SAR test reports. Each numerical smartphone model comprised a smartphone platform and an antenna. Although many components and materials are used in commercial smartphones, we simplified the numerical smartphone platform by considering only the essential parts; hence, the numerical smartphone platform consisted of a casing, liquid-crystal display (LCD) glass, LCD dielectrics, an LCD ground, a metal chassis, a main board, and a battery. The thickness of the battery was $3 \mathrm{~mm}$ and the thickness of the remaining components was $1 \mathrm{~mm}$. Fig. 1 shows a schematic diagram of the numerical smartphone platform. Note that the length of all components was set to an integer multiple of $1 \mathrm{~mm}$ to avoid overwhelming FDTD simulation burdens. This smartphone platform was used throughout the research. We designed various antennas and integrated them into the smartphone platform to develop the final numerical smartphone models.

Based on the previously mentioned SAR test reports, we considered LTE Band 1 (1,920-1,930 MHz), LTE Band 3 (1,715$1,785 \mathrm{MHz})$, LTE Band 5 (824-849 $\mathrm{MHz})$, LTE Band 7 (2,500-2,570 MHz), LTE Band 8 (905-915 MHz), a wideband code division multiple access (WCDMA) band (1,922.81,977.2 MHz), and a WLAN band (2,412-2,472 MHz). The development of seven numerical smartphone models for each frequency band was unnecessary; hence, for this study, we developed four numerical smartphone models by grouping similar frequency bands together because their peak SAR values were almost identical for small frequency deviations, as demonstrated in [9]. Table 1 shows the four frequency bands used for this study, with their corresponding SAR target frequencies.

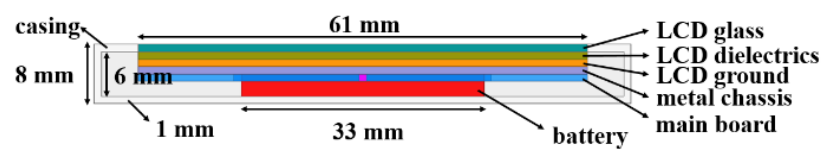

(a)

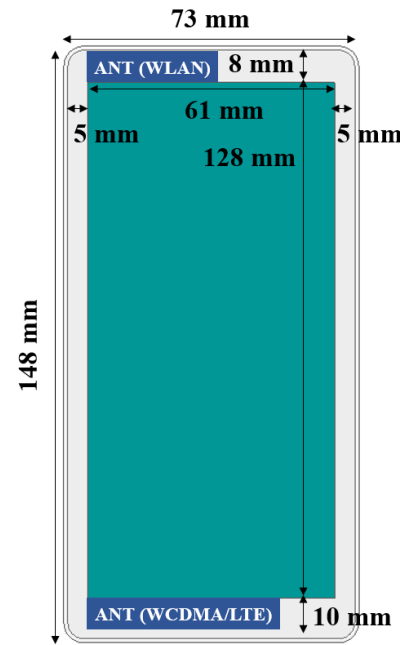

(b)

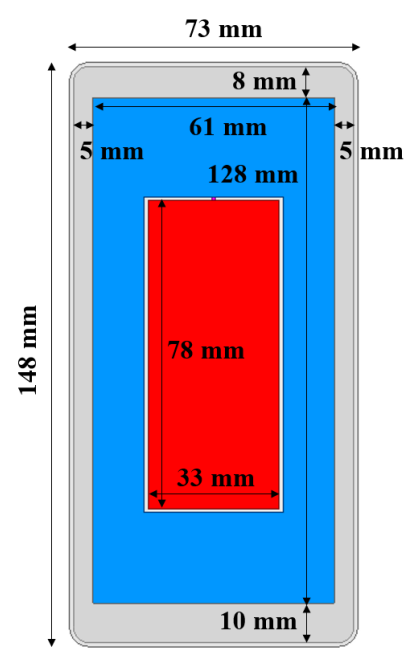

(c)
Fig. 1. Schematic diagram of the numerical smartphone platform: (a) side view, (b) front view, and (c) back view.

Table 1. Frequency band categorization

\begin{tabular}{lcc}
\hline \multicolumn{1}{c}{ Band } & Bandwidth $(\mathrm{MHz})$ & $\begin{array}{c}\text { SAR target frequency } \\
(\mathrm{MHz})\end{array}$ \\
\hline WCDMA/LTE B1/B3 & $1,715-1,980$ & 1,850 \\
LTE B5/B8 & $824-915$ & 870 \\
LTE B7 & $2,500-2,570$ & 2,535 \\
WLAN & $2,412-2,472$ & 2,450 \\
\hline
\end{tabular}

Next, we determined the antenna locations based on the SAR test reports. Most antennas for the WCDMA and LTE bands were located at the bottom, whereas the WLAN antennas were mostly located at the top left; therefore, we determined the positions of the antennas integrated into the numerical smartphone models as bottom and top left for the WCDMA/LTE bands and the WLAN band, respectively, as shown in Fig. 1(b).

SAR values were evaluated for four positions-left cheek, left tilt, right cheek, and right tilt - as shown in Fig. 2. The cheek position indicates that a smartphone is in contact with the cheek along the reference plane, and the tilt position indicates a smartphone tilt of $15^{\circ}$ against the cheek position.

For this study, we used the SAM phantom described in the IEC/IEEE international standard [16]. Table 2 lists the electrical properties of the SAM phantom. For target frequencies not specified by [16], the electrical properties were obtained using the linear interpolation technique. We now address the FDTD cell modeling for the SAR study for the tilt positions in detail. 


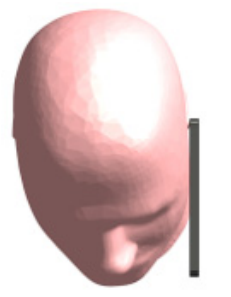

(a)

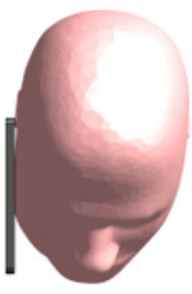

(b)

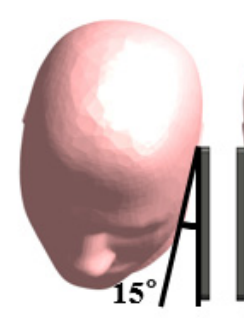

(c)

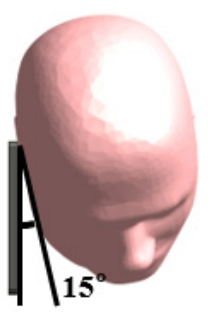

(d)
Fig. 2. Smartphone positions for simulated SAM phantoms:

(a) left cheek, (b) right cheek, (c) left tilt, and (d) right tilt.

Table 2. Electrical properties of the SAM phantom

\begin{tabular}{ccc}
\hline $\begin{array}{c}\text { SAR target frequency } \\
(\mathrm{MHz})\end{array}$ & Relative permittivity & Conductivity (S/m) \\
\hline 1,850 & 40 & 1.4 \\
870 & 41.5 & 0.9385 \\
2,535 & 39.0845 & 1.8935 \\
2,450 & 39.2 & 1.8 \\
\hline
\end{tabular}

The FDTD method uses orthogonal cells; thus, geometrical models that are not parallel to the major $(x, y$, and $z)$ coordinates suffer from staircasing errors. For tilt positions, we should choose to rotate the numerical smartphone model or the SAM phantom by $15^{\circ}$. The structure of the numerical smartphone model was significantly more complicated than that of the SAM phantom. When tilting the numerical smartphone model, the resulting staircasing errors reduced FDTD accuracy more severely than did the SAM phantom rotation; therefore, we positioned the numerical smartphone model along the major coordinates and tilted the SAM phantom (see Fig. 2).

The target $1-\mathrm{g}$ psSAR values were determined by averaging the 1-g psSAR values of the smartphones for each frequency band in Table 1. As mentioned previously, most WLAN antennas are located on the top left. In this work, to consider as many WLAN antennas as possible, WLAN antennas on the top right were also included to average the 1 -g psSAR values using geometrical symmetry; for example, the 1-g psSAR values of the left cheek position for the top-right antennas were used to obtain the target 1-g psSAR values for the right cheek position. In the same fashion, the $1-\mathrm{g}$ psSAR values of the right cheek position for the top-right antennas were employed to obtain the target 1-g psSAR values of the left cheek position. A similar procedure was utilized for the tilt positions.

As stated previously, the aim of this work was to develop computational models for numerical SAR research on smartphone use. We designed antennas for the four categorized frequency bands to finalize the numerical smartphone models. Antennas should be designed so that the 1-g psSAR values of numerical smartphone models are as close to the target 1-g
psSAR values as possible. Moreover, antennas should yield good performance for their matching and radiation characteristics. In this work, we designed antennas so that the numerical smartphone models satisfied the following criteria:

(1) The $S_{11}$ parameter is less than $-6 \mathrm{~dB}$, with or without the SAM phantom.

(2) The radiation efficiency is higher than $70 \%$ without the SAM phantom.

(3) The difference in the 1 -g psSAR value is within $\pm 20 \%$.

Fig. 3 summarizes the design flowchart for the numerical smartphone models.

In this work, we designed an inverted-F antenna (IFA) for a numerical smartphone platform, the configuration of which is shown in Fig. 4. According to the design procedure described above, we optimized the geometrical parameters for each antenna for the four frequency bands, as listed in Table 3.

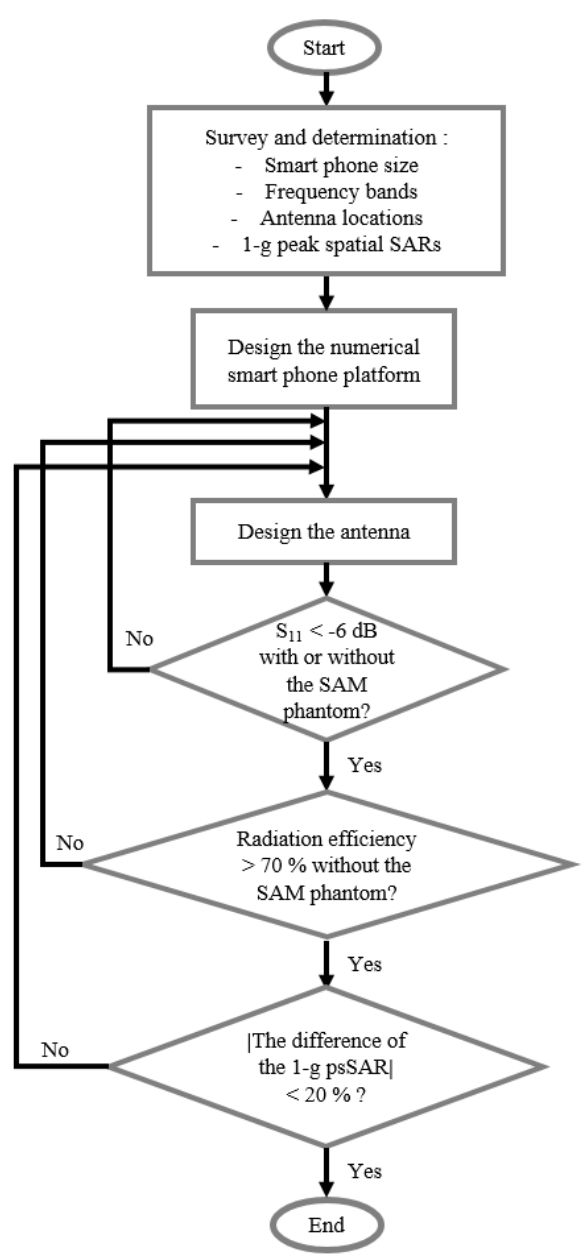

Fig. 3. Design flowchart for numerical smartphone models.

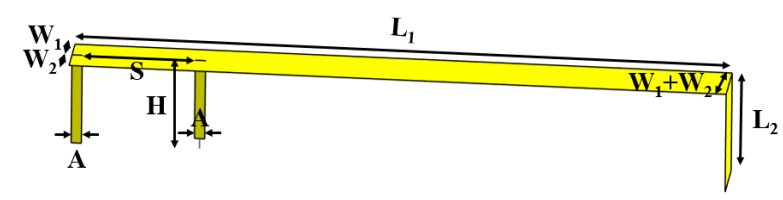

Fig. 4. IFA configuration. 
Table 3. Antenna parameters for each frequency band (unit: $\mathrm{mm}$ )

\begin{tabular}{lccccccc}
\hline \multicolumn{1}{c}{ Band } & $\mathrm{W}_{1}$ & $\mathrm{~W}_{2}$ & $\mathrm{~S}$ & $\mathrm{H}$ & $\mathrm{L}_{1}$ & $\mathrm{~L}_{2}$ & $\mathrm{~A}$ \\
\hline WCDMA/LTE B1/ B3 & 3 & 3 & 3 & 4 & 28 & - & 1 \\
LTE B5/B8 & 2 & - & 11 & 9 & 64 & 16 & 1 \\
LTE B7 & - & 3 & 20 & 6 & 38 & - & 1 \\
WLAN & 1 & - & 2 & 4 & 28 & - & 1 \\
\hline
\end{tabular}

\section{RESULTS FOR THE NUMERICAL SMARTPHONE}

\section{MODELS}

This section presents the simulation results for the numerical smartphone models for the four categorized frequency bands. Fig. 5 shows that all $S_{11}$ parameters of the designed numerical

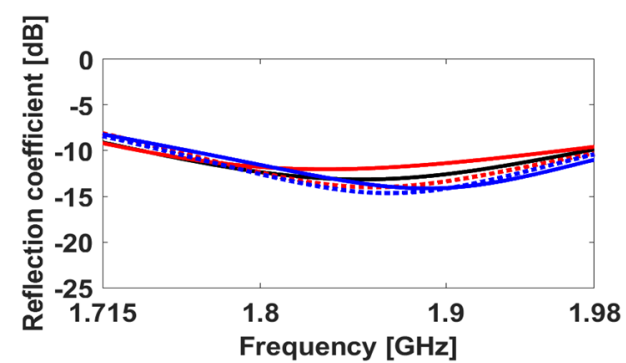

(a)

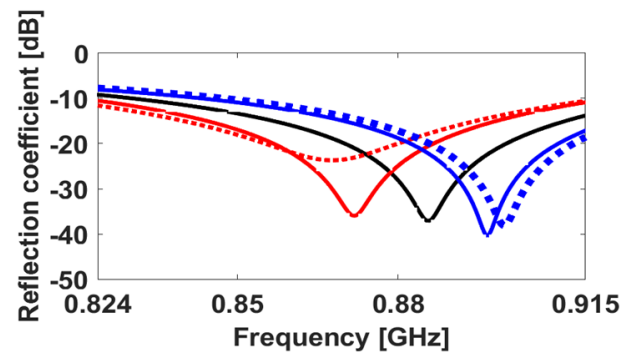

(b)

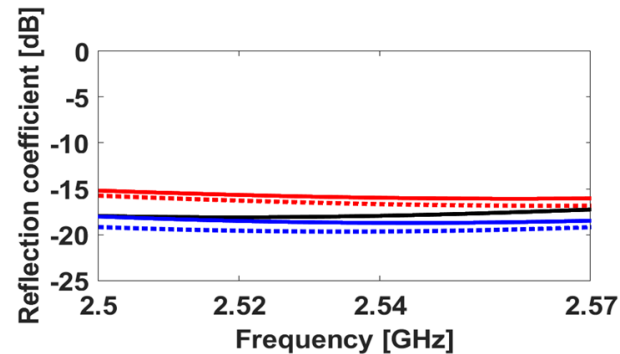

(c)

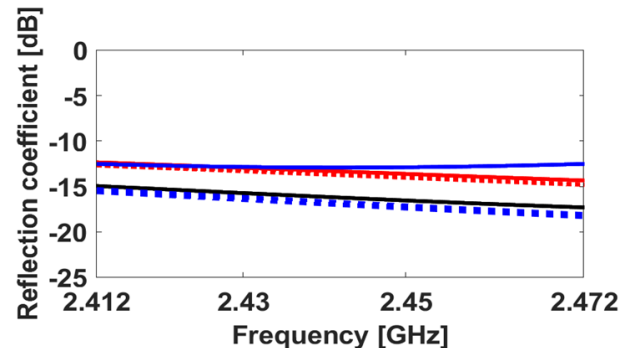

(d)

Fig. 5. Reflection coefficients: (a) WCDMA/LTE B1/B3, (b) LTE B5/B8, (c) LTE B7, and (d) WLAN. smartphone models were below $-7.2 \mathrm{~dB}$, with or without the SAM phantom. Fig. 6 shows the efficiencies of the numerical smartphone models. As shown in Fig. 6, the radiation efficiencies of the designed phone models without the SAM phantom exceeded $78 \%$. For the SAM phantom, the radiation efficiency decreased due to the EM absorption of the SAM phantom, as expected. Specifically, the radiation efficiency in the tilt positions was always larger than in the cheek positions because the distance between the antenna and the SAM phantom increased in the tilt positions more than in the cheek positions. Also, for the SAM phantom, the radiation efficiency for the WLAN band was worse than for the WCDMA/LTE bands. As mentioned previously, the WLAN antenna was located on the top left of the smartphone model, and the WCDMA/LTE antennas were located at the bottom; therefore, the SAM phantom

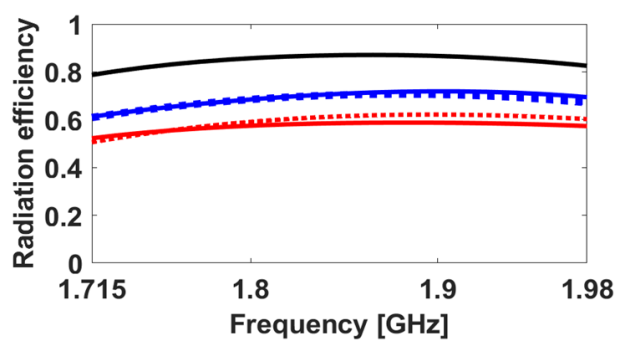

(a)

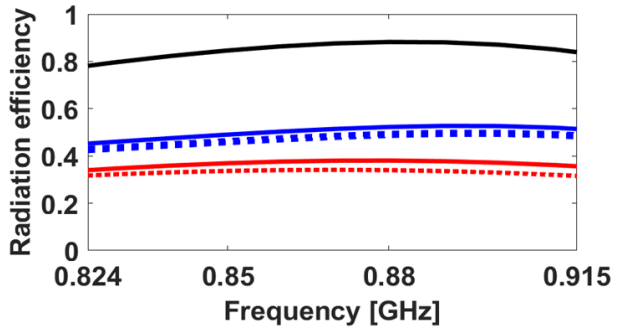

(b)

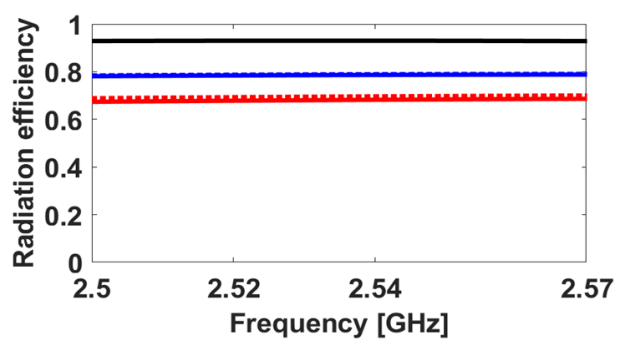

(c)

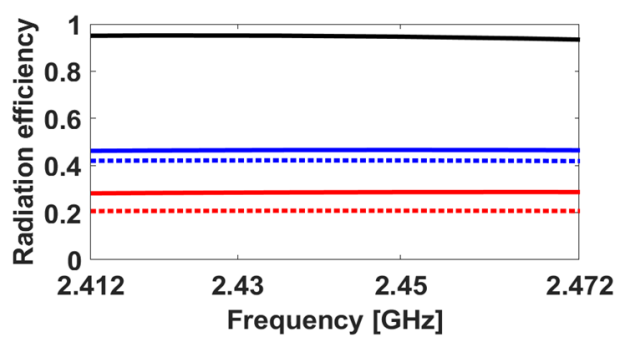

(d)

Fig. 6. Radiation efficiency: (a) WCDMA/LTE B1/B3, (b) LTE B5/B8, (c) LTE B7, and (d) WLAN. 
decreased the radiation efficiency for the WLAN more severely than for the other cases.

Next, we simulated the 1-g psSAR values for the numerical smartphone models. The difference values were determined by comparing the simulated 1-g psSAR values with the target 1-g psSAR values, as listed in Tables 4-7. As shown in Table 4, the largest differences in the simulated $1-\mathrm{g}$ psSAR values versus the target value were $\pm 14.89 \%$ in the cheek positions. Table 5 shows that the largest difference between the simulated 1-g psSAR value and the target value was $-12.77 \%$ in the left tilt

Table 4. Target and result values for the 1-g psSAR for WCDMA/LTE B1/B3 at an output power of $54 \mathrm{~mW}$

\begin{tabular}{lccccc}
\hline & \multicolumn{2}{c}{ Cheek position } & & \multicolumn{2}{c}{ Tilt position } \\
\cline { 2 - 3 } \cline { 5 - 6 } & Left & Right & & Left & Right \\
\hline Target value (W/kg) & 0.188 & 0.188 & & 0.107 & 0.107 \\
Result value (W/kg) & 0.160 & 0.216 & & 0.119 & 0.105 \\
Difference (\%) & -14.89 & 14.89 & & 11.21 & -1.87 \\
\hline
\end{tabular}

Table 5. Target and result values for the 1-g psSAR for LTE B5/B8 at an output power of $33.9 \mathrm{~mW}$

\begin{tabular}{lccccc}
\hline & \multicolumn{2}{c}{ Cheek position } & & \multicolumn{2}{c}{ Tilt position } \\
\cline { 2 - 3 } \cline { 6 - 6 } & Left & Right & & Left & Right \\
\hline Target value $(\mathrm{W} / \mathrm{kg})$ & 0.166 & 0.166 & & 0.094 & 0.094 \\
Result value $(\mathrm{W} / \mathrm{kg})$ & 0.158 & 0.186 & & 0.082 & 0.091 \\
Difference $(\%)$ & -4.82 & 12.05 & & -12.77 & -3.19 \\
\hline
\end{tabular}

Table 6. Target and result values for the 1-g psSAR for LTE B7 at an output power of $34.2 \mathrm{~mW}$

\begin{tabular}{lccccc}
\hline & \multicolumn{2}{c}{ Cheek position } & & \multicolumn{2}{c}{ Tilt position } \\
\cline { 2 - 3 } \cline { 5 - 6 } & Left & Right & & Left & Right \\
\hline Target value (W/kg) & 0.135 & 0.135 & & 0.070 & 0.070 \\
Result value (W/kg) & 0.145 & 0.125 & & 0.081 & 0.059 \\
Difference (\%) & 7.41 & -7.41 & & 15.71 & -15.71 \\
\hline
\end{tabular}

Table 7. Target and result values for the $1-\mathrm{g}$ psSAR for WLAN at an output power of $8.64 \mathrm{~mW}$

\begin{tabular}{lccccc}
\hline & \multicolumn{2}{c}{ Cheek position } & & \multicolumn{2}{c}{ Tilt position } \\
\cline { 2 - 3 } \cline { 5 - 6 } & Left & Right & & Left & Right \\
\hline Target value (W/kg) & 0.194 & 0.221 & & 0.155 & 0.199 \\
Result value (W/kg) & 0.165 & 0.254 & & 0.145 & 0.189 \\
Difference (\%) & -14.95 & 14.93 & & -6.45 & -5.03 \\
\hline
\end{tabular}

position for LTE Band 5 and LTE Band 8. For LTE Band 7, the largest differences in the simulated 1-g psSAR values were $\pm 15.71 \%$ in the tilt positions. In the WLAN band, the largest difference between the simulated $1-\mathrm{g}$ psSAR value and the target value was $-14.95 \%$ in the left cheek position. All differences in the 1-g psSAR values for the numerical smartphone models met the design criterion $( \pm 20 \%)$. For the numerical smartphone models with the antenna at the bottom, the $1-\mathrm{g}$ psSAR values in the cheek positions were higher than in the tilt positions, as shown in Tables 4-6. When the antenna is located at the bottom of the phone, the ground current intensity is strong near the bottom or center of the phone ground; therefore, the distance between the SAM phantom and the phone ground (on which the strong current flows) plays a crucial role in SAR evaluation. This distance in the cheek positions is shorter than in the tilt positions; therefore, when the antenna is located at the bottom of the phone, higher 1-g psSAR values are obtained in the cheek positions versus the tilt positions, as illustrated in [17].

It is worth noting that, in the numerical smartphone models, we did not include shielding materials or components for SAR reduction, which were usually used in commercial smartphones; therefore, the output power levels of numerical smartphone models are lower than those of commercial smartphones. Again, it should be stressed that our $1-\mathrm{g}$ psSAR values for the four different SAR test positions at a certain output power satisfied the design criterion.

\section{CONCLUSION}

Previous epidemiological studies were confined to mobile phones for the $2 \mathrm{G}$ and $3 \mathrm{G}$ communication services. In this work, for ongoing epidemiological studies, we proposed numerical modeling of smartphones for LTE and WLAN services. Based on the SAR test reports for smartphones released in South Korea in 2013-2019, we determined the smartphone size, antenna locations, frequency band categorization, and target 1-g psSAR values. We developed the numerical smartphone models using the following design criteria: First, the return loss of the numerical smartphone models should be larger than $6 \mathrm{~dB}$, regardless of SAM existence. Second, the radiation efficiency of the numerical smartphone models without the SAM phantom should exceed 70\%. Finally, the differences between the simulated 1-g psSAR values of the smartphone models for the four SAR test positions and the target values should be within $\pm 20 \%$. The numerical results showed that the designed smartphone models satisfied all of the above-mentioned criteria. We believe that our numerical smartphone models can be successfully employed in future epidemiological studies to investigate the health risks of smartphone use. 
This work was supported in part by the ICT R\&D program of MSIT/IITP (No. 2019-0-00102, A Study on Public Health and Safety in a Complex EMF Environment) and in part by the National Research Foundation of Korea (NRF) grant funded by the Korea government (MSIT) (No. 2020R1F1A1055444). The authors would like to thank Samsung Electronics for allowing the use of SAR test reports.

\section{REFERENCES}

[1] E. Cardis, L. Richardson, I. Deltour, B. Armstrong, M. Feychting, C. Johansen, et al., "The INTERPHONE study: design, epidemiological methods, and description of the study population," European Journal of Epidemiology, vol. 22, no. 9, pp. 647-664, 2007.

[2] D. Aydin, M. Feychting, J. Schuz, T. Tynes, T. V. Andersen, L. S. Schmidt, et al., "Mobile phone use and brain tumors in children and adolescents: a multicenter case: control study," Journal of the National Cancer Institute, vol. 103, no. 16, pp. 1264-1276, 2011.

[3] J. Schuz, P. Elliott, A. Auvinen, H. Kromhout, A. H. Poulsen, C. Johansen, et al., "An international prospective cohort study of mobile phone users and health (Cosmos): design considerations and enrolment," Cancer Epidemiology, vol. 35, no. 1, pp. 37-43, 2011.

[4] S. Sadetzki, C. E. Langer, R. Bruchim, M. Kundi, F. Merletti, R. Vermeulen, et al., "The MOBI-Kids study protocol: challenges in assessing childhood and adolescent exposure to electromagnetic fields from wireless telecommunication technologies and possible association with brain tumor risk," Frontiers in Public Health, vol. 2, article no. 124, 2014. https://doi.org/10.3389/fpubh.2014.00124

[5] J. Wiart, A. Hadjem, M. F. Wong, and I. Bloch, "Analysis of RF exposure in the head tissues of children and adults," Physics in Medicine \& Biology, vol. 53, no. 13, pp. 3681-3695, 2008.

[6] A. Christ, M. C. Gosselin, M. Christopoulou, S. Kuhn, and N. Kuster, "Age-dependent tissue-specific exposure of cell phone users," Physics in Medicine \& Biology, vol. 55, no. 7, pp. 1767-1783, 2010.

[7] A. K. Lee, S. E. Hong, J. H. Kwon, H. D. Choi, and E. Cardis, "Mobile phone types and SAR characteristics of the human brain," Physics in Medicine \& Biology, vol. 62, no. 7, pp. 2741-2761, 2017.

[8] A. K. Lee, J. S. Park, S. E. Hong, M. Taki, K. Wake, J. Wiart, and H. D. Choi, "Brain SAR of average male Korean child to adult models for mobile phone exposure assessment," Physics in Medicine \& Biology, vol. 64, no. 4, article no. 045004, 2019. https://doi.org/10.1088/1361-6560/aafcdc

[9] A. K. Lee, Y. Yoon, S. Lee, B. Lee, S. E. Hong, H. D. Choi, and E. Cardis, "Numerical implementation of representative mobile phone models for epidemiological studies," Journal of Electromagnetic Engineering and Science, vol. 16, no. 2, pp. 87-99, 2016.

[10] Sim4life: a revolutionary simulation platform [Online]. Available: http://zurichmedtech.com.

[11] A. Taflove and S. C. Hagness, Computational Electromagnetics: The Finite-Difference Time-Domain Method, 3rd ed. Norwood, MA: Artech House, 2005.

[12] R. A. Chilton, K. Y. Jung, R. Lee, and F. L. Teixeira, "Frozen modes in parallel-plate waveguides loaded with magnetic photonic crystals," IEEE Transactions on Microwave Theory and Techniques, vol. 55, no. 12, pp. 2631-2641, 2007.

[13] H. Choi, J. W. Baek, and K. Y. Jung, "Comprehensive study on numerical aspects of modified Lorentz modelbased dispersive FDTD formulations," IEEE Transactions on Antennas and Propagation, vol. 67, no. 12, pp. 76437648, 2019.

[14] H. Choi, J. W. Baek, and K. Y. Jung, "Numerical stability and accuracy of CCPR-FDTD for dispersive media," IEEE Transactions on Antennas and Propagation, vol. 68, no. 11, pp. 7717-7720, 2000.

[15] Y. J. Kim and K. Y. Jung, "Accurate and efficient finitedifference time-domain formulation of dusty plasma," IEEE Transactions on Antennas and Propagation, vol. 69, no. 10, pp. 6600-6606, 2021.

[16] Measurement procedure for the assessment of specific absorption rate of human exposure to radio frequency fields from hand-held and body-mounted wireless communication devices - Part 1528: Human models, instrumentation, and procedures (Frequency range of $4 \mathrm{MHz}$ to $10 \mathrm{GHz}$ ), IEEE/IEC 62209-1528-2020, 2020.

[17] J. Lee, S. Park, A. K. Lee, H. D. Choi, and K. Y. Jung, "Numerical study of maximum peak spatial SAR reduction in a mobile phone," Journal of Electrical Engineering \& Technology, vol. 15, no. 5, pp. 2211-2216, 2020. 
Jisu Lee

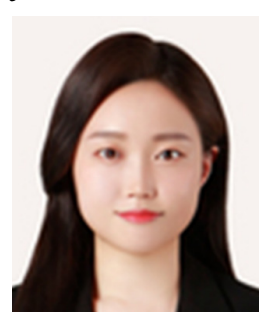

received her B.S. degree in Electronic Engineering from Seokyeong University, Seoul, Republic of Korea, in 2019. She is currently working toward a Ph.D. in electronic engineering at Hanyang University, Seoul, Republic of South Korea. Her current research interests include antenna development.

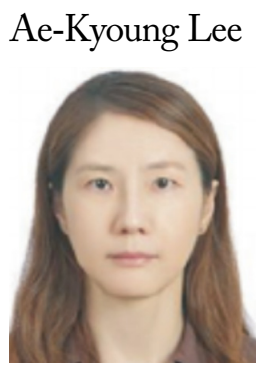

received her B.S. and M.S. degrees in electronic engineering from Chungang University, Seoul, Republic of Korea in 1990 and 1992, respectively, and a Ph.D. in radio science and engineering from Chungnam National University, Daejeon, Republic of South Korea in 2003. In 1992, she joined the Radio Technology Group at the Electronics and Telecommunications Research Institute, Daejeon, Republic of Korea, where she is currently a principal researcher and has been involved in projects on measurement technologies and numerical analyses of electromagnetic compatibility and human exposure to RF fields. She was the recipient of the Japan Microwave Prize at the 1998 AsiaPacific Microwave Conference, Japan, and the Technology Award from the Korean Institute of Electromagnetic Engineering and Science in 1999.

Seon-Eui Hong

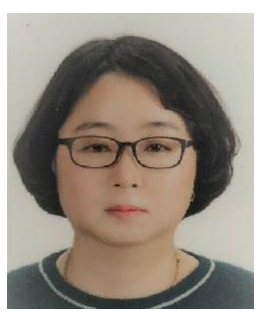

received her M.S. and Ph.D. degrees in radio science and engineering from Chungnam National University, Daejeon, Republic of Korea, in 1999 and 2017, respectively. Since 1999, she has been with the Electronics and Telecommunications Research Institute, Daejeon, Republic of Korea, and is currently a principal member of the radio environment and monitoring research group. Her current research interests include numerical dosimetry and procedures for assessing an electromagnetic source.

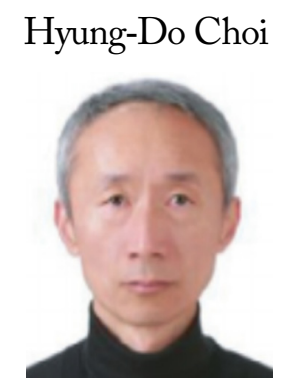

received his M.S. and Ph.D. degrees in material science from Korea University, Seoul, Republic of Korea, in 1989 and 1996, respectively. Since 1997, he has been with the Electronics and Telecommunications Research Institute, Republic of South Korea. He has performed research on the biological effects of $\mathrm{RF}$ radiation and has developed $\mathrm{RF}$ radiation protection standards and regulations. His current research interests include spectrum management, microwave tomography, and EMC countermeasures.

\section{Kyung-Young Jung}

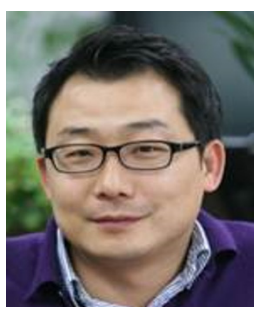

received his B.S. and M.S. degrees in electrical engineering from Hanyang University, Seoul, Republic of Korea, in 1996 and 1998, respectively, and his $\mathrm{Ph} . \mathrm{D}$. in electrical and computer engineering from Ohio State University, Columbus, USA, in 2008. From 2008 to 2009, he was a postdoctoral researcher at Ohio State University, and from 2009 to 2010, he was an assistant professor in the Department of Electrical and Computer Engineering, Ajou University, Suwon, Republic of South Korea. Since 2011, he has worked at Hanyang University, where he is now a professor in the Department of Electronic Engineering. His current research interests include computational electromagnetics, bioelectromagnetics, and nanoelectromagnetics. Dr. Jung received a Graduate Study Abroad Scholarship from the National Research Foundation of Korea, a Presidential Fellowship from Ohio State University, a HYU Distinguished Teaching Professor Award from Hanyang University, and an Outstanding Research Award from the Korean Institute of Electromagnetic Engineering and Science. 